
\title{
R Reserach S Suare \\ Validity and Reliability of Handgrip Dynamometry in Older Adults: A Comparison of Two Widely Used Dynamometers
}

\author{
Melissa J. Benton ( $\square$ mbenton@uccs.edu ) \\ University of Colorado at Colorado Springs https://orcid.org/0000-0001-8616-3350 \\ Jefferson M. Spicher \\ University of Colorado Colorado Springs \\ Amy L. Silva-Smith \\ University of Colorado Colorado Springs
}

Research article

Keywords: Aging, Frailty, Sarcopenia, Handgrip strength, Jamar dynamometer, Smedley dynamometer

Posted Date: May 10th, 2021

DOI: https://doi.org/10.21203/rs.3.rs-500869/v1

License: (c) (i) This work is licensed under a Creative Commons Attribution 4.0 International License.

Read Full License 


\section{Abstract \\ Background}

Among older adults, decreased handgrip strength is associated with greater risk of frailty, and loss of physical function, mobility, lean mass, and overall muscular strength and power. Frailty is also associated with sarcopenia, for which handgrip strength has been recommended for diagnostic purposes. Specific cutoff points for diagnosis have been identified but use of different devices may affect measurement. Therefore to assess validity and reliability, we compared Jamar hydraulic versus Smedley spring handgrip dynamometers.

\section{Methods}

Sixty-seven older $(76.2 \pm 0.9$ years $)$ men $(n=34)$ and women $(n=33)$ completed two trials of handgrip strength measurement on sequential days $(\mathrm{T} 1, \mathrm{~T} 2)$ using both devices in random order. Intraclass correlations were used to assess test-retest reliability, and Bland-Altman analysis was used to assess validity as the level of agreement between devices.

\section{Results}

There were significant $(p<0.001)$ relationships between devices at T1 $(r=0.94)$ and T2 $(r=0.94)$ and strong $(p<0.001)$ intraclass correlations were observed for both devices (Jamar $=0.98$; Smedley $=0.96)$, indicating excellent reliability. However, there were significant differences between devices. Strength measured with Jamar was greater than Smedley at both T1 $(27.4 \pm 1.4 \mathrm{vs} .23 .4 \pm 1.1 \mathrm{~kg}, p<0.001)$ and T2 $(25.3 \pm 1.4$ vs. $21.8 \pm 1.2 \mathrm{~kg}, p<0.001)$. Bland-Altman analysis confirmed these differences. Subgroup analysis to evaluate the effect of gender and age indicated that in women and old-old ( $>75$ years) participants, differences between devices were closer to zero for both measurements compared to men and young-old (65-75 years) participants.

\section{Conclusions}

Our results demonstrate that despite excellent reliability, there is poor agreement between devices, indicating a lack of validity. For use as a diagnostic tool, standardization and device-specific cut points for handgrip dynamometry are needed.

\section{Background}

In middle and older-aged adults, handgrip strength predicts all-cause and disease-specific mortality, including mortality related to cardiovascular disease, chronic obstructive pulmonary disease, and cancer [1-7]. Furthermore, among older adults in particular, decreased handgrip strength is associated with 
greater risk of frailty, and loss of physical function, mobility, lean mass, and overall muscular strength and power [8-14]. Handgrip strength is generally recognized as a surrogate measure of whole-body strength and can be used clinically to assess for age-related deterioration in function and health status associated with frailty $[3,8,14]$.

Frailty and loss of function and health are also associated with sarcopenia, a geriatric syndrome characterized by loss of muscle and strength [15]. Globally, the prevalence of sarcopenia among adults aged 60 years and over is estimated to be at least 10\% [16]. Sarcopenia not only predicts mortality among community-dwelling and acutely ill older adults [17-19], but is also related to functional decline, loss of independence, and hospitalization [20-22]. Exercise interventions can successfully prevent and reverse muscle loss and functional decline $[23,24]$, but clinical assessment is needed to identify older adults who are at risk [16].

Muscle strength is a biomarker for sarcopenia [25], and handgrip strength measured with dynamometry has been recommended for diagnostic purposes $[26,27]$. However, although absolute and precise genderspecific cut points for normal handgrip strength have been identified, these cut points do not consider potential differences between measurement devices. Currently, there is no universally agreed-upon device or procedure for clinical measurement [26, 27]. In fact, a systematic review of handgrip measurement protocols found incomplete reporting of both the procedures and the devices used [28]. The Jamar hydraulic dynamometer is widely used, but multiple other devices are available for clinical and research purposes [29]. Recent systematic reviews of handgrip strength named at least 10 different devices used for measurement $[4,6,30]$. Among these, the Jamar hydraulic dynamometer and Smedley spring dynamometer were the most frequently identified $[4,6,30]$.

There are similarities and differences between the two dynamometers. Both devices weigh approximately $0.66 \mathrm{~kg}$ and provide force measurements up to $90 \mathrm{~kg}$. However, the Jamar hydraulic dynamometer displays force using an analog dial with 2-kilogram increments, so smaller more discrete measurements must be interpreted by the operator. By comparison, the Smedley uses a digital display that provides force measurements to the nearest $0.1 \mathrm{~kg}$, so operator interpretation is eliminated. Also, both have adjustable handles, to modify grip size, although the Jamar has a concave grip while the Smedley grip is straight. Finally, the Jamar is metal, so the surface temperature can be cooler to touch than the Smedley, which is plastic.

The differences in these two devices may influence the validity and reliability of measurement. To date, we can find only one study comparing the Jamar and Smedley dynamometers in older adults [31]. Although measurements obtained by the two devices were similar, they were statistically different and influenced by gender and age. Specifically, differences were greater in women compared to men, and in older compared to younger participants [31]. Moreover, only a single trial was used for comparison, so reliability over time could not be evaluated. Therefore, to assess validity and reliability, we compared sequential grip strength measurements in older adults over a two-day period using a Jamar hydraulic (Patterson Medical, USA) versus a Smedley spring (Takei Scientific Instruments, Japan) handgrip 
dynamometer. Our secondary aim was to evaluate the effect of gender and age on agreement between devices.

\section{Methods \\ Design}

The current study was part of a larger study that has previously been described [32]. Briefly, this was an empirical $2 \times 2$ design. Participants completed two measurement sessions on consecutive days (T1, T2) using two devices (Jamar, Smedley). T1 measurements were scheduled in the middle of the day when participants were normally fed and hydrated. T2 measurements were scheduled in the early morning on the following day when participants were fasting (i.e., without food or fluids for at least eight hours). This design was specifically intended to elicit loss of muscular strength and function between T1 and T2, which allowed the researchers to assess both reliability and validity when measurements changed over time. The order of testing for each device was randomized between participants and between times. Although interrater reliability for handgrip dynamometry is good to excellent [33], all data were obtained by the same investigator to avoid any potential differences. Ethical approval was obtained from the University of Colorado Colorado Springs Institutional Review Board and all participants signed an informed consent prior to enrollment.

\section{Participants}

Sixty-seven community-dwelling older adults $(76.2 \pm 0.9$ years) volunteered and completed both measurement sessions. Inclusion criteria were age 65 years or older, non-smoking, and able to stand up and ambulate independently or with an assistive device. The only exclusion criterion was the inability to hold the dynamometer and maintain correct positioning during measurement. No participants were excluded from the study.

\section{Measurements}

\section{Anthropometrics}

Body measurements were obtained using standardized procedures [34]. Before measurement, participants were asked to void and remove their shoes and all excess clothing. Weight was calculated to the nearest $0.2 \mathrm{~kg}$. Height and waist circumference were calculated to the nearest $0.5 \mathrm{~cm}$.

\section{Handgrip Dynamometry}

For all measurements, the grip width on the Jamar was standardized to the second position $(5.0 \mathrm{~cm})$ that has been found to maximize strength production in the majority of adults regardless of age, body mass, or hand dimensions $[35,36]$. The grip width of the Smedley was also adjusted to $5.0 \mathrm{~cm}$ for uniformity between devices. Consistent with recommendations for handgrip dynamometry by the American Society of Hand Therapy and previous research [10,31], participants sat in a chair with the device held in their 
dominant hand, their arm supported on a table or other stable surface, their wrist in a neutral position, and their elbow bent at a $90^{\circ}$ angle. This procedure has been reported to have high test-retest reliability [37]. Participants then squeezed the device one time, as hard as possible, for 3 seconds. A single attempt was used for each device to avoid muscle fatigue and loss of strength attributed to multiple attempts [36, $38,39]$. After a 2-minute rest, participants repeated the same measurement procedure with the second device. The maximal force exerted with the Smedley was measured to the nearest $0.1 \mathrm{~kg}$ using the digital readout. The maximal force exerted with the Jamar was measured to the nearest $2.0 \mathrm{~kg}$ using markers on the analog dial and then estimated by the investigator to the nearest $0.5 \mathrm{~kg}$ based on visual inspection of the gauge needle's position between the 2-kg markers.

\section{Statistical Analysis}

Data were analyzed using SPSS version 27.0 (IBM Corporation, USA) and reported as mean \pm SE with $95 \% \mathrm{Cl}$ unless otherwise indicated. Statistical significance was set as $p<0.05$. Analysis of variance (ANOVA) was used to assess differences at T1 and T2. Pearson correlations were used to evaluate the association between the two devices at T1 and T2, and intraclass correlations (ICC) were used to assess test-retest reliability. For purposes of this analysis, values between $0.8-0.9$ were considered good, and values above 0.9 were considered excellent [40]. Bland-Altman analysis was used to assess the level of agreement between devices by plotting differences \pm 2 SD against mean values [41]. Plots were visually assessed for characteristics demonstrating good agreement, including mean values close to zero, uniform distribution over the range of measurement, and $95 \%$ of differences within \pm 2 SD [41]. Finally, to examine the effect of gender and age on agreement, data were stratified by age (young-old 65-75 years, old-old $>75$ years) and gender (male, female).

\section{Results}

\section{Participant Characteristics}

Thirty-four men and 33 women (age range 65-96 years) completed the study. There were no differences between men and women for age or body mass index (BMI). However, males had significantly greater ( $p$ $<0.001$ ) height, body weight, waist circumference and handgrip strength (Table 1). 
Table 1

Participant characteristics

\begin{tabular}{|c|c|c|c|}
\hline & $\begin{array}{l}\text { All } \\
(N=67)\end{array}$ & $\begin{array}{l}\text { Men } \\
(n=34)\end{array}$ & $\begin{array}{l}\text { Women } \\
(n=33)\end{array}$ \\
\hline \multirow[t]{2}{*}{ Age (years) } & $76.2 \pm 0.9$ & $75.8 \pm 1.2$ & $76.7 \pm 1.2$ \\
\hline & {$[74.5,77.9]$} & {$[73.4,78.2]$} & {$[74.1,79.2]$} \\
\hline \multirow[t]{2}{*}{ Height (cm) } & $170.8 \pm 1.3$ & $179.3 \pm 1.0 *$ & $162.0 \pm 1.1$ \\
\hline & {$[168.2,173.4]$} & {$[177.2,181.4]$} & {$[159.8,164.2]$} \\
\hline \multirow[t]{2}{*}{ Weight (kg) } & $76.0 \pm 1.9$ & $83.9 \pm 2.4^{*}$ & $67.9 \pm 2.2$ \\
\hline & {$[72.3,79.8]$} & {$[79.1,88.8]$} & {$[63.5,72.3]$} \\
\hline \multirow[t]{2}{*}{ Body mass index $\left(\mathrm{kg} / \mathrm{m}^{2}\right)$} & $26.0 \pm 0.5$ & $26.1 \pm 0.7$ & $25.9 \pm 0.8$ \\
\hline & {$[25.0,27.0]$} & {$[24.7,27.4]$} & {$[24.3,27.5]$} \\
\hline \multirow[t]{2}{*}{ Waist circumference $(\mathrm{cm})$} & $96.5 \pm 1.7$ & $102.5 \pm 1.9 *$ & $90.2 \pm 2.3$ \\
\hline & {$[93.1,99.8]$} & {$[98.7,106.3]$} & {$[85.5,95.0]$} \\
\hline Hand grip strength (kg) & $27.4 \pm 1.4 \mp \S$ & $34.1 \pm 2.0 *$ & $20.6 \pm 1.1$ \\
\hline T1 Jamar & {$[24.7,30.2]$} & {$[30.1,38.0]$} & {$[18.4,22.8]$} \\
\hline \multirow[t]{2}{*}{ T1 Smedley } & $23.4 \pm 1.1 \ddagger$ & $29.1 \pm 1.5^{*}$ & $17.4 \pm 0.9$ \\
\hline & {$[21.1,25.6]$} & {$[26.1,32.2]$} & {$[15.5,19.3]$} \\
\hline Hand grip strength (kg) & $25.3 \pm 1.4 \S$ & $31.5 \pm 2.1^{*}$ & $18.8 \pm 1.0$ \\
\hline T2 Jamar & {$[22.5,28.1]$} & {$[27.4,35.7]$} & {$[16.7,20.9]$} \\
\hline \multirow[t]{2}{*}{ T2 Smedley } & $21.8 \pm 1.2$ & $27.4 \pm 1.6^{*}$ & $16.1 \pm 1.0$ \\
\hline & {$[19.5,24.1]$} & {$[24.1,30.6]$} & {$[14.1,18.0]$} \\
\hline \multicolumn{4}{|c|}{ Data presented as mean \pm SE $[95 \% \mathrm{Cl}]$} \\
\hline \multicolumn{4}{|c|}{ *Significant difference between Men and Women $(p<0.001)$} \\
\hline \multicolumn{4}{|c|}{ \#Significant difference between T1 and T2 $(p<0.001)$} \\
\hline \multicolumn{4}{|c|}{ §Significant difference between Jamar and Smedley $(p<0.001)$} \\
\hline
\end{tabular}

\section{Reliability}

There were significant within-group differences between T1 and T2 for both devices (Table 1). For the Jamar, strength decreased 8\% $(p<0.001)$ and for the Smedley, strength decreased $7 \%(p<0.001)$. Despite these differences, both devices were positively correlated (T1: $r=0.94, p<0.001 ; \mathrm{T} 2: r=0.94, p<0.001$ ) (Fig. 1) and test-retest reliability was excellent (ICC: Jamar $=0.98, p<0.001$; Smedley $=0.92, p<0.001$ ). 


\section{Validity}

There were significant between-group differences between devices at both T1 and T2 (Table 1). At T1, there was an average $( \pm S D)$ difference of $4.1 \pm 4.2 \mathrm{~kg}(p<0.001)$ between the Jamar and Smedley dynamometers, and at T2 the average ( \pm SD) difference was $3.5 \pm 4.0 \mathrm{~kg}(p<0.001)$ (Table 2). BlandAltman analysis indicated poor agreement between the Jamar and Smedley dynamometers at T1 and T2 (Fig. 2). Mean values were not close to zero, although they were closer at T2 than at T1, and distribution was not uniform over the range of either measurement. Also, although $97 \%$ of differences fell within \pm 2 $\mathrm{SD}$ at $\mathrm{T} 1$, only $94 \%$ of differences fell within \pm 2 SD at $\mathrm{T} 2$.

Table 2

Differences in grip strength between the Jamar and Smedley dynamometers at $\mathrm{T} 1$ and $\mathrm{T} 2$.

\begin{tabular}{|lll|}
\hline & $\begin{array}{l}\text { Difference at T1 } \\
\text { Mean } \pm \text { SD } \\
{[95 \% \mathrm{Cl}]}\end{array}$ & $\begin{array}{l}\text { Difference at T2 } \\
\text { Mean } \pm \text { SD } \\
{[95 \% \mathrm{Cl}]}\end{array}$ \\
\hline All participants $(\mathrm{N}=67)$ & $4.1 \pm 4.2$ & $3.5 \pm 4.0$ \\
& {$[3.1,5.1]$} & {$[2.5,4.4]$} \\
\hline Men $(\mathrm{n}=34)$ & $4.9 \pm 4.9$ & $4.2 \pm 4.5$ \\
\hline Women $(n=33)$ & $3.3 \pm 3.2$ & {$[2.6,5.8]$} \\
\hline Young-Old $(n=33)$ & $4.9 \pm 4.3$ & $2.7 \pm 3.2$ \\
& {$[3.4,6.4]$} & $4.7 \pm 4.4$ * \\
\hline Old-Old $(n=34)$ & $3.3 \pm 4.0$ & {$[3.2,6.3]$} \\
\hline *Significant difference between Young-Old and Old-Old $(p=0.009)$ \\
\hline
\end{tabular}

\section{Effect of Gender and Age}

When data were stratified by gender, mean differences were not statistically different between men and women at either $\mathrm{T} 1$ or $\mathrm{T} 2$, indicating no effect for gender, although differences in women were closer to zero for both measurements (Table 2). Visual inspection of Bland-Altman plots found women to cluster at the lower end of the range of strength measurement, reflecting lower absolute grip strength, but with generally similar differences between devices as those observed for men (Fig. 3). Interestingly, all differences falling outside of \pm 2 SD from mean values were for men. 
When data were stratified by age, mean differences were not statistically different at $\mathrm{T} 1$, indicating no effect of age on initial measurement. However, at T2 the mean difference between devices in young-old participants was statistically greater than the mean difference in old-old participants (Table 2), indicating a possible age effect. Visual inspection of Bland-Altman plots found differences in old-old participants to cluster somewhat closer to zero along the range, reflecting smaller mean differences (Fig. 4). Clustering was more evident at $\mathrm{T} 2$, consistent with the statistically significant difference from young-old participants for that measurement.

\section{Discussion}

To our knowledge, this is the first study to evaluate both the validity and reliability of the Jamar and Smedley handgrip dynamometers that are the most widely used dynamometers for research purposes. Significant differences in handgrip strength were observed between devices at both timepoints, indicating poor validity that was confirmed with visual inspection of Bland Altman plots. However, intra-class correlations for both devices were excellent, indicating good reliability for both devices.

Previously, Guerra and Amaral [31] compared the Jamar and Smedley dynamometers at one time point only in a sample of 55 older adults between 65-99 years of age. They reported a correlation coefficient of $r=0.83$ with a mean difference of $3.2 \mathrm{~kg}$, which is similar to but slightly smaller than our current findings. In contrast to our findings, they reported that the level of agreement between the two devices was poorer for women compared to men, and old-old compared to young-old. In our sample, women demonstrated better agreement than men, and old-old participants demonstrated better agreement than the young-old. Participant differences may somehow have influenced discrepancies in our findings. Our sample was somewhat younger, with an average age of 76.2 years, compared to 79.2 years for their sample. Furthermore, our sample was evenly distributed between men and women, while theirs was predominantly $(76 \%)$ female.

Guerra and Amaral [31] attributed discrepancies in measurement to an interaction between participants and dynamometer characteristics. As previously described, there are both similarities and differences between the Jamar and Smedley dynamometers. Although they may be considered subtle, these differences could be sufficient to cause the discrepancies in measurement observed in both our studies, while differences in our samples may have been sufficient to cause the inconsistent effects of gender and age between our studies. For example, the ability to exert handgrip strength is influenced by pain or discomfort [13], so the design of each dynamometer may be of importance. More research in this area is needed.

It has been suggested that increasing handgrip strength may independently improve physical and functional resilience, resulting in improved health outcomes [2]. A meta-analysis of 25 studies, including almost 200,000 adults with an average age of 65 years or older, demonstrates that increasing handgrip strength by only $1 \mathrm{~kg}$ reduces mortality due to heart disease by $30 \%$ [6]. This is an intriguing finding, but 
without clear guidelines for handgrip strength measurement, accurate clinical assessment is difficult and appropriate implementation of interventions is problematic.

Current diagnostic criteria for sarcopenia in older adults differentiate handgrip strength cut points based on gender [26,27]. This is supported by the absolute differences between men and women we observed. However, the diagnostic cut points ignore differences between devices that are highlighted by our current findings. Specific cut points of $<27 \mathrm{~kg}$ for men and $<16 \mathrm{~kg}$ for women are recommended by the European Working Group on Sarcopenia in Older People (EWGSOP) [26], and $<26 \mathrm{~kg}$ for men and $<18 \mathrm{~kg}$ for women are recommended by the Asian Working Group for Sarcopenia (AWGS) [27], but they are not specific to the device used for measurement. By comparison to handgrip strength, the AWGS has now differentiated cut points for muscle mass that are device specific [27]. They differentiate cutoff values for skeletal muscle based on either dual-energy $x$-ray absorptiometry (DXA) or bioelectrical impedance analysis (BIA), in recognition that both are widely used technologies with numerous advantages but without absolute agreement. Based on our findings, we believe that device-specific cut points for handgrip strength are also needed. This is consistent with the EWGSOP's key recommendation that simple, specific cutoff points for measures used to identify sarcopenia be developed [26].

We recognize that there are limitations to our study. Our sample size was relatively small, although it exceeded the sample used for the only previous study we could find that compared the Jamar and Smedley dynamometers in older adults. Furthermore, a small sample is primarily of concern due to the risk of a type II error. The significant differences we observed appear to rule out this concern in relation to our findings. It is also possible that use of another procedure for handgrip measurement may have obviated differences between the two devices. As previously noted, there are multiple procedures reported in the literature that include variations in arm positioning and the number of attempts used for measurement. The influence of these variations should be explored in future research.

\section{Conclusion}

The mean differences we observed between the Jamar and Smedley, two of the most widely used handgrip dynamometers, could result in misdiagnosis either for or against sarcopenia (i.e., either a false positive or a false negative). Nevertheless, as a diagnostic tool, handgrip dynamometry has numerous advantages including low cost, portability, rapid results, and easy use. However, without device-specific cut points or a universally agreed-upon device for handgrip dynamometry, sarcopenia treatment and research may be impeded. Individual labs and clinics may not have universal access to all types of devices available, so device-specific cut points for handgrip dynamometry would appear to have the greater advantage and are recommended.

\section{Abbreviations}

AWGS: Asian Working Group for Sarcopenia; BIA: Bioelectrical impedance analysis; BMI: body mass index; DXA: dual-energy x-ray absorptiometry; EWGSOP: European Working Group on Sarcopenia in Older 
People

\section{Declarations}

\section{Acknowledgements}

Not applicable.

\section{Authors' contributions}

MJB, JMS, and ALS were all involved in designing the study and recruiting participants. MJB was responsible for data collection and analysis, and preparation of the draft manuscript. MJB, JMS, and ALS all contributed to critical revisions and approved the final version of the manuscript.

\section{Availability of data and materials}

The datasets used and analyzed for the current study are available from the corresponding author on reasonable request.

\section{Competing interests}

All authors declare they have no competing interests.

\section{Consent for publication}

Not applicable.

\section{Ethics approval and consent to participate}

Ethical approval was obtained from the University of Colorado Colorado Springs Institutional Review Board (protocol \# 14-132) and all participants signed an informed consent prior to enrollment.

\section{Funding}

No funding was received for this study.

\section{References}

1. Celis-Morales CA, Welsh P, Lyall DM, Steell L, Petermann F, Anderson J, et al. Associations of grip strength with cardiovascular, respiratory, and cancer outcomes and all cause mortality: prospective cohort study of half a million UK Biobank participants. BMJ. 2018;361:k1651.

2. Rantanen T, Harris T, Leveille SG, Visser M, Foley D, Masaki K, et al. Muscle strength and body mass index as long-term predictors of mortality in initially healthy men. J Gerontol A Biol Sci Med Sci. 2000;55(3):M168-73. 
3. Rantanen T, Volpato S, Ferrucci L, Heikkinen E, Fried LP, Guralnik JM. Handgrip strength and causespecific and total mortality in older disabled women: exploring the mechanism. J Am Geriatr Soc. 2003;51(5):636-41.

4. Rijk JM, Roos PR, Deckx L, van den Akker M, Buntinx F. Prognostic value of handgrip strength in people aged 60 years and older: A systematic review and meta-analysis. Geriatr Gerontol Int. 2016;16(1):5-20.

5. Gale CR, Martyn CN, Cooper C, Sayer AA. Grip strength, body composition, and mortality. Int J Epidemiol. 2007;36(1):228-35.

6. Lee J. Associations Between Handgrip Strength and Disease-Specific Mortality Including Cancer, Cardiovascular, and Respiratory Diseases in Older Adults: A Meta-Analysis. J Aging Phys Act. 2020;28(2):320-31.

7. Chung CJ, Wu C, Jones M, Kato TS, Dam TT, Givens RC, et al. Reduced handgrip strength as a marker of frailty predicts clinical outcomes in patients with heart failure undergoing ventricular assist device placement. J Card Fail. 2014;20(5):310-5.

8. Alonso AC, Ribeiro SM, Luna NMS, Peterson MD, Bocalini DS, Serra MM, et al. Association between handgrip strength, balance, and knee flexion/extension strength in older adults. PLoS One. 2018;13(6):e0198185.

9. Wisniowska-Szurlej A, Cwirlej-Sozanska A, Woloszyn N, Sozanski B, Wilmowska-Pietruszynska A. Association between Handgrip Strength, Mobility, Leg Strength, Flexibility, and Postural Balance in Older Adults under Long-Term Care Facilities. Biomed Res Int. 2019;2019:1042834.

10. Sherwood JJ, Inouye C, Webb SL, O J. Reliability and Validity of the Sit-to-Stand as a Muscular Power Measure in Older Adults. J Aging Phys Act. 2020;28:455-66.

11. Rantanen T, Era P, Kauppinen M, Heikkinen E. Maximal Isometric Muscle Strength and Socioeconomic Status, Health, and Physical Activity in 75-Year-Old Persons. Journal of Aging and Physical Activity. 1994;2:206-20.

12. Gopinath B, Kifley A, Liew G, Mitchell P. Handgrip strength and its association with functional independence, depressive symptoms and quality of life in older adults. Maturitas. 2017;106:92-4.

13. Payette H, Hanusaik N, Boutier V, Morais JA, Gray-Donald K. Muscle strength and functional mobility in relation to lean body mass in free-living frail elderly women. Eur J Clin Nutr. 1998;52(1):45-53.

14. Fried LP, Tangen CM, Walston J, Newman AB, Hirsch C, Gottdiener J, et al. Frailty in older adults: evidence for a phenotype. J Gerontol A Biol Sci Med Sci. 2001;56(3):M146-56.

15. McPhee JS, Cameron J, Maden-Wilkinson T, Piasecki M, Yap MH, Jones DA, et al. The Contributions of Fiber Atrophy, Fiber Loss, In Situ Specific Force, and Voluntary Activation to Weakness in Sarcopenia. J Gerontol A Biol Sci Med Sci. 2018;73(10):1287-94.

16. Shafiee G, Keshtkar A, Soltani A, Ahadi Z, Larijani B, Heshmat R. Prevalence of sarcopenia in the world: a systematic review and meta- analysis of general population studies. J Diabetes Metab Disord. 2017; 16:21. 
17. Chang SF, Lin PL. Systematic Literature Review and Meta-Analysis of the Association of Sarcopenia With Mortality. Worldviews Evid Based Nurs. 2016;13(2):153-62.

18. Vetrano DL, Landi F, Volpato S, Corsonello A, Meloni E, Bernabei R, et al. Association of sarcopenia with short- and long-term mortality in older adults admitted to acute care wards: results from the CRIME study. J Gerontol A Biol Sci Med Sci. 2014;69(9):1154-61.

19. Liu P, Hao Q, Hai S, Wang H, Cao L, Dong B. Sarcopenia as a predictor of all-cause mortality among community-dwelling older people: A systematic review and meta-analysis. Maturitas. 2017;103:1622.

20. Batsis JA, Mackenzie TA, Lopez-Jimenez F, Bartels SJ. Sarcopenia, sarcopenic obesity, and functional impairments in older adults: National Health and Nutrition Examination Surveys 19992004. Nutr Res. 2015;35(12):1031-9.

21. Beaudart C, Zaaria M, Pasleau F, Reginster JY, Bruyere O. Health Outcomes of Sarcopenia: A Systematic Review and Meta-Analysis. PLoS One. 2017;12(1):e0169548.

22. Bjorkman M, Jyvakorpi SK, Strandberg TE, Pitkala KH, Tilvis RS. Sarcopenia Indicators as Predictors of Functional Decline and Need for Care among Older People. J Nutr Health Aging. 2019;23(10):91622.

23. Vlietstra L, Hendrickx W, Waters DL. Exercise interventions in healthy older adults with sarcopenia: A systematic review and meta-analysis. Australas J Ageing. 2018;37(3):169-83.

24. Yoshimura Y, Wakabayashi H, Yamada M, Kim H, Harada A, Arai H. Interventions for Treating Sarcopenia: A Systematic Review and Meta-Analysis of Randomized Controlled Studies. J Am Med Dir Assoc. 2017;18(6):553 e1- e16.

25. Cesari M, Fielding RA, Pahor M, Goodpaster B, Hellerstein M, van Kan GA, et al. Biomarkers of sarcopenia in clinical trials-recommendations from the International Working Group on Sarcopenia. $J$ Cachexia Sarcopenia Muscle. 2012;3(3):181-90.

26. Cruz-Jentoft AJ, Bahat G, Bauer J, Boirie Y, Bruyere O, Cederholm T, et al. Sarcopenia: revised European consensus on definition and diagnosis. Age Ageing. 2019;48(1):16-31.

27. Chen LK, Liu LK, Woo J, Assantachai P, Auyeung TW, Bahyah KS, et al. Sarcopenia in Asia: consensus report of the Asian Working Group for Sarcopenia. J Am Med Dir Assoc. 2014;15(2):95101.

28. Sousa-Santos AR, Amaral TF. Differences in handgrip strength protocols to identify sarcopenia and frailty - a systematic review. BMC Geriatr. 2017;17(1):238.

29. Innes E. Handgrip strength testing: A review of the literature. Australian Occupational Therapy Journal. 1999;46(3):120-40.

30. Bohannon RW. Test-Retest Reliability of Measurements of Hand-Grip Strength Obtained by Dynamometry from Older Adults: A Systematic Review of Research in the PubMed Database. J Frailty Aging. 2017;6(2):83-7.

31. Guerra RS, Amaral TF. Comparison of hand dynamometers in elderly people. J Nutr Health Aging. 2009;13(10):907-12. 
32. Benton MJ, Silva-Smith AL, Spicher JM. Muscle loss is associated with risk of orthostatic hypotension in older men and women. The Journal of Frailty \& Aging. 2020;In Press.

33. Vanpee G, Hermans G, Segers J, Gosselink R. Assessment of limb muscle strength in critically ill patients: a systematic review. Crit Care Med. 2014;42(3):701-11.

34. Lohman TG, Martorell R, Roche AF. Anthropometric Standardization Reference Manual. Champaign, IL: Human Kinetics; 1988.

35. Firrell JC, Crain GM. Which setting of the dynamometer provides maximal grip strength? J Hand Surg Am. 1996;21(3):397-401.

36. Crosby CA, Wehbe MA, Mawr B. Hand strength: normative values. J Hand Surg Am. 1994;19(4):66570.

37. Bohannon RW, Bubela DJ, Magasi SR, Gershon RC. Relative reliability of three objective tests of limb muscle strength. Isokinet Exerc Sci. 2011;19(2).

38. Abizanda P, Navarro JL, Garcia-Tomas MI, Lopez-Jimenez E, Martinez-Sanchez E, Paterna G. Validity and usefulness of hand-held dynamometry for measuring muscle strength in community-dwelling older persons. Arch Gerontol Geriatr. 2012;54(1):21-7.

39. Reijnierse EM, de Jong N, Trappenburg MC, Blauw GJ, Butler-Browne G, Gapeyeva H, et al. Assessment of maximal handgrip strength: how many attempts are needed? J Cachexia Sarcopenia Muscle. 2017;8(3):466-74.

40. Koo TK, Li MY. A Guideline of Selecting and Reporting Intraclass Correlation Coefficients for Reliability Research. J Chiropr Med. 2016;15(2):155-63.

41. Bland JM, Altman DG. Statistical methods for assessing agreement between two methods of clinical measurement. Lancet. 1986;1(8476):307-10.

\section{Figures}



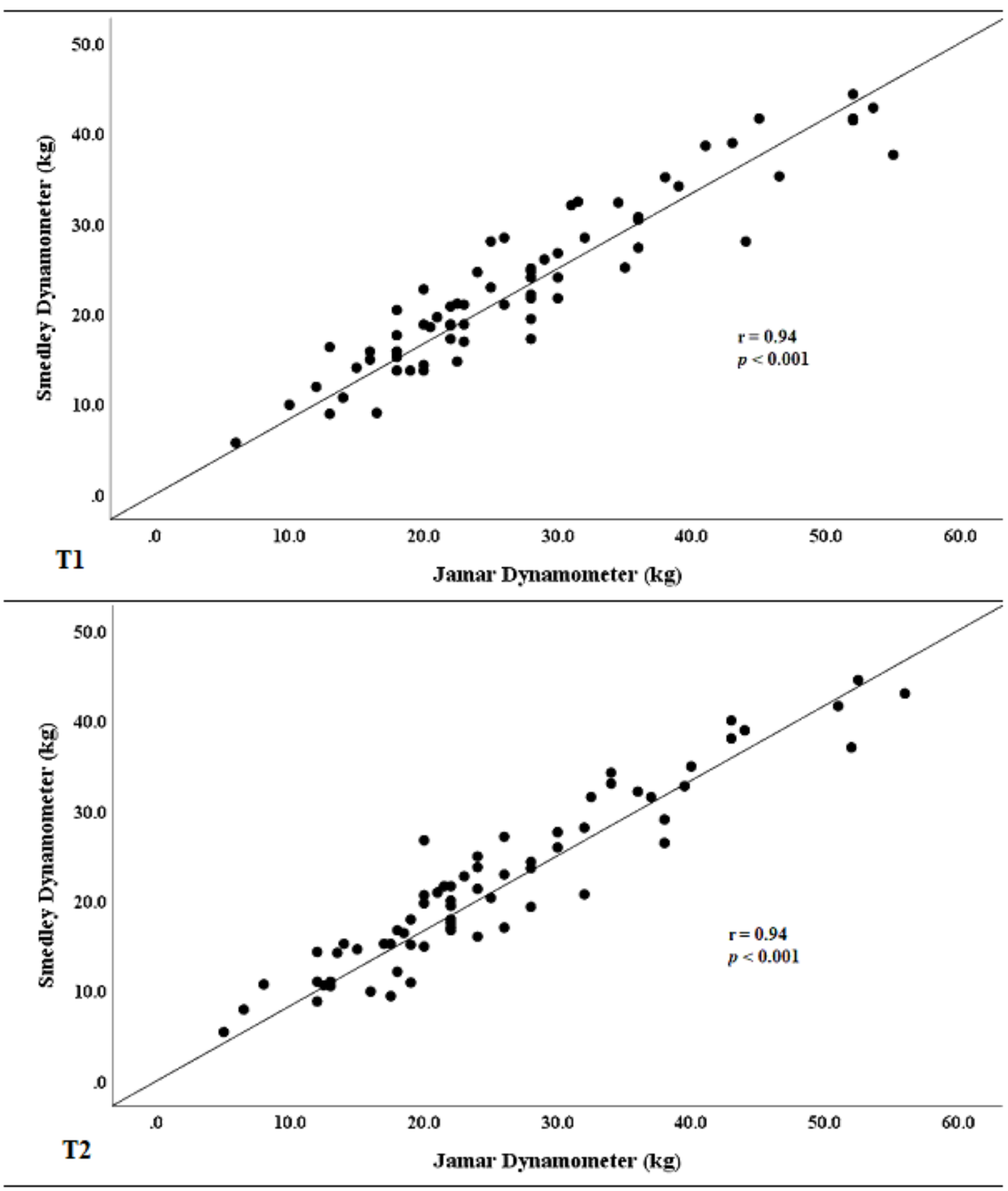

Figure 1

Correlation between devices at T1 (top) and T2 (bottom). Hand grip measurements were strongly and positively correlated at both timepoints. 


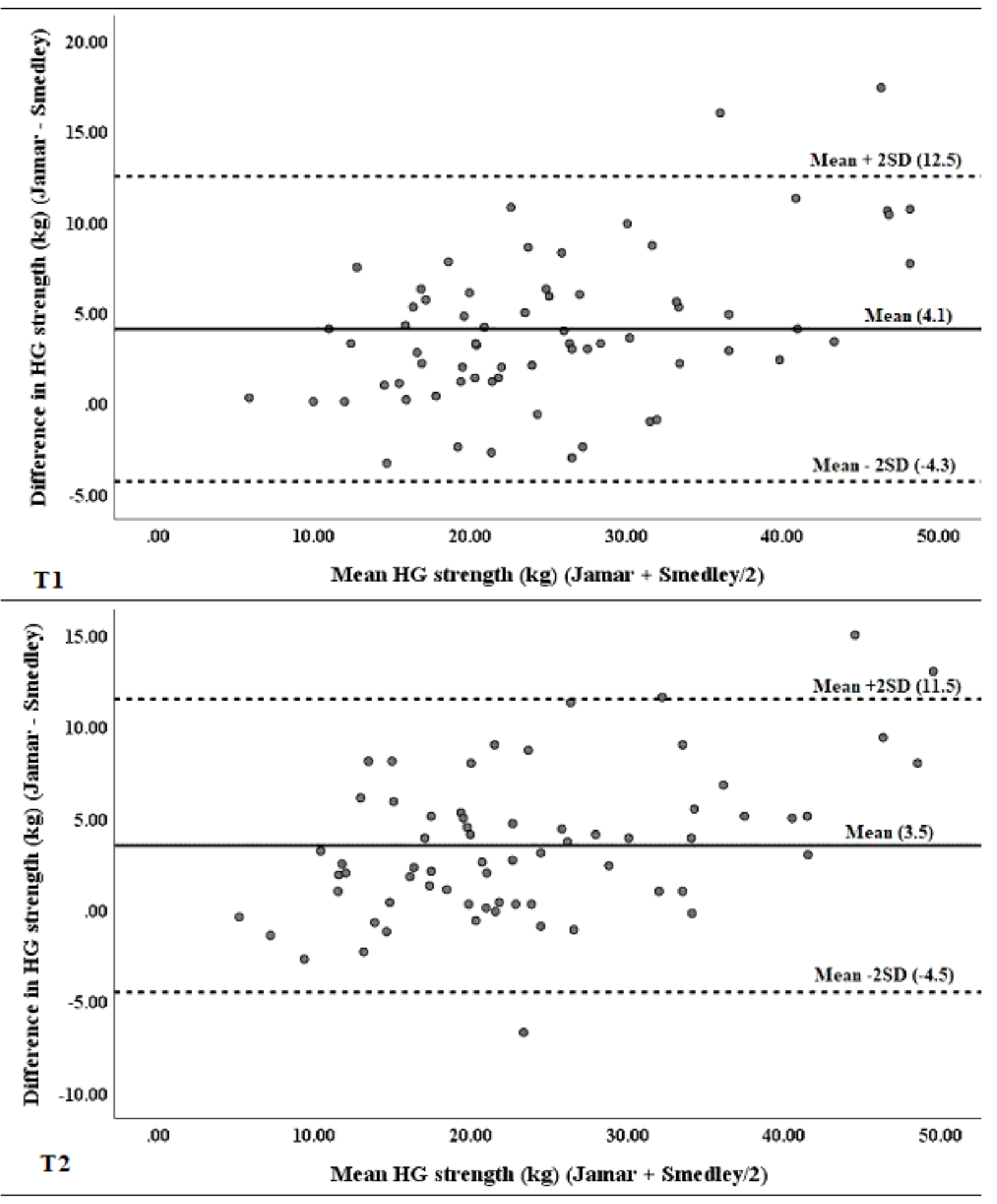

Figure 2

Bland-Altman plots for differences against mean values of hand grip strength measured with the Jamar and Smedley dynamometers at T1 (top) a,nd T2 (bottom). 


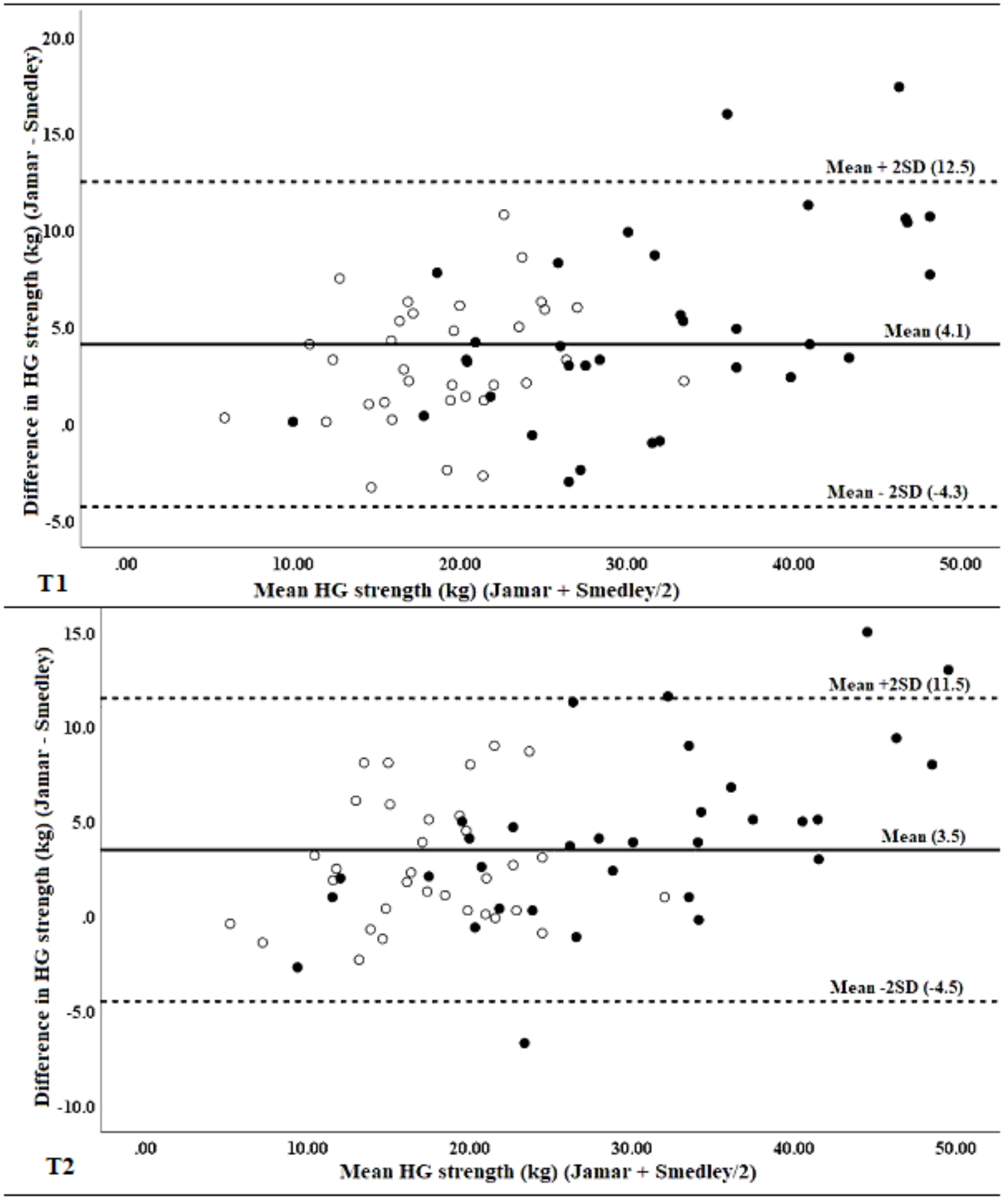

Figure 3

Bland-Altman plots for differences against mean values of hand grip strength measured with the Jamar and Smedley dynamometers at T1 (top) and T2 (bottom) stratified by gender (women = empty circles, men $=$ solid circles) . 


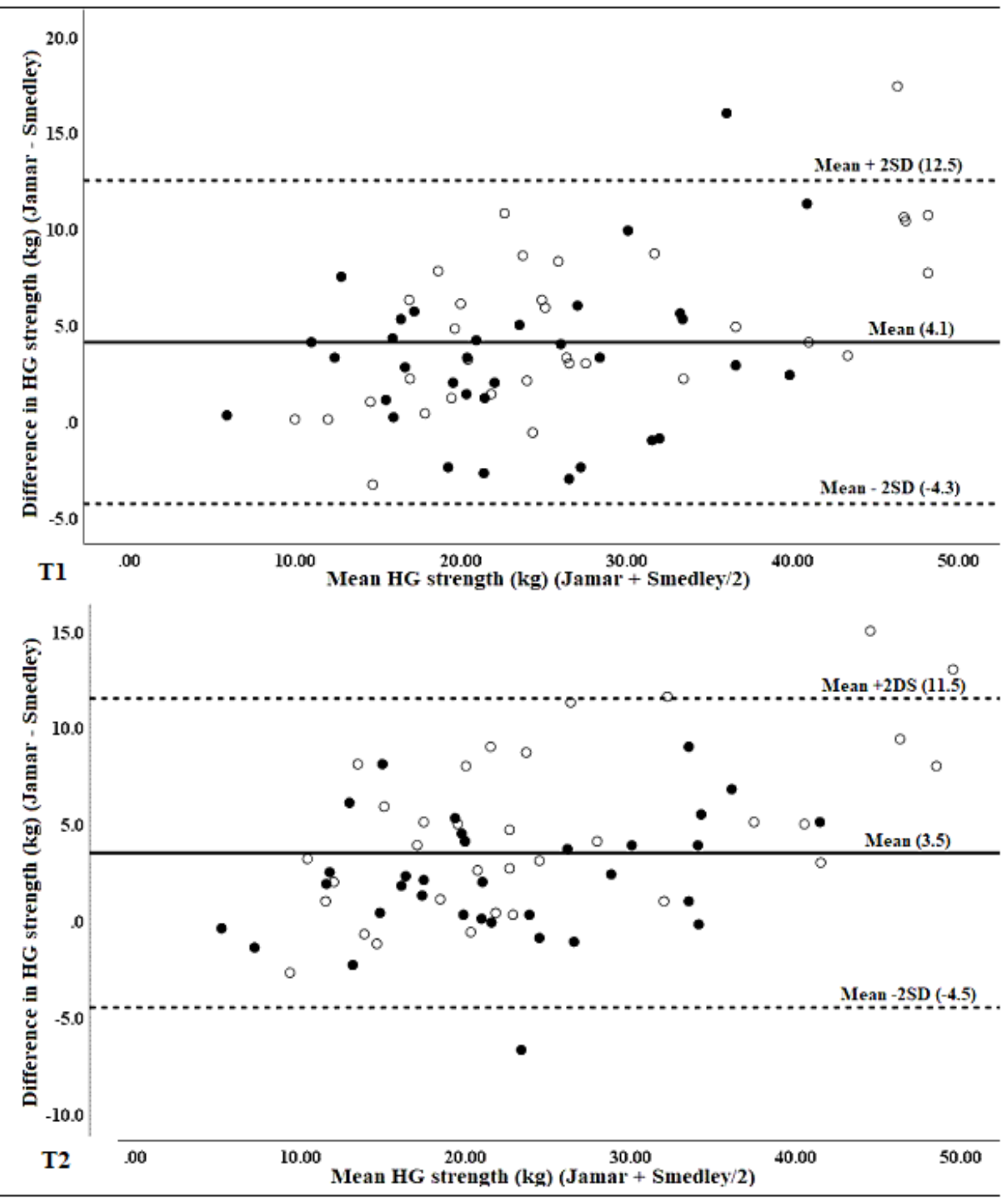

Figure 4

Bland-Altman plots for differences against mean values of hand grip strength measured with the Jamar and Smedley dynamometers at T1 (top) and T2 (bottom) stratified by age (young-old = empty circles, oldold = solid circles). 